\title{
Solution of a functional equation on compact groups using Fourier analysis
}

\begin{abstract}
Let $G$ be a compact group, let $n \in \mathbb{N} \backslash\{0,1\}$ be a fixed element and let $\sigma$ be a continuous automorphism on $G$ such that $\sigma^{n}=I$. Using the non-abelian Fourier transform, we determine the non-zero continuous solutions $f: G \rightarrow \mathbb{C}$ of the functional equation
\end{abstract}

$$
f(x y)+\sum_{k=1}^{n-1} f\left(\sigma^{k}(y) x\right)=n f(x) f(y), \quad x, y \in G,
$$

in terms of unitary characters of $G$.

1. Introduction. Let $G$ be a group, let $n \in \mathbb{N} \backslash\{0,1\}$ be a fixed element and let $\sigma$ be an automorphism on $G$ such that $\sigma^{n}=I$, where $I$ denotes the identity map. We consider the functional equation

$$
f(x y)+\sum_{k=1}^{n-1} f\left(\sigma^{k}(y) x\right)=n f(x) f(y), \quad x, y \in G,
$$

where $f: G \rightarrow \mathbb{C}$ is the function to determine. This equation, in the case where $G$ is abelian, has been studied by many authors (see, e.g., Shin'ya [14, Corollary 3.12] and Stetkær [18, Theorem 14.9]). Eq. (1.1) is a generalization of the following variant of d'Alembert's functional equation

$$
f(x y)+f(\sigma(y) x)=2 f(x) f(y), \quad x, y \in G,
$$

2010 Mathematics Subject Classification. 39B52, 22C05, 43A30, $22 \mathrm{E} 45$.

Key words and phrases. Functional equation, non-abelian Fourier transform, representation of a compact group. 
which was introduced and solved on semigroups by Stetkær in [20]. Some information, applications and numerous references concerning (1.2), d'Alembert's functional equation

$$
f(x+y)+f(x-y)=2 f(x) f(y), \quad x, y \in \mathbb{R},
$$

and their further generalizations can be found e.g. in ([1]-[12]; [15]-[22]).

The purpose of the present paper is to solve the functional equation (1.1) in the case where $G$ is a compact group and possibly non-abelian. Our approach uses the harmonic analysis and the representation theory on compact groups. We note that the idea of using Fourier analysis for solving (1.1) goes back to [5].

Throughout the rest of this paper, $G$ is a compact group with identity element $e$ and $\sigma$ is a continuous automorphism on $G$ such that $\sigma^{n}=I$. By solutions (resp. representations), we always mean continuous solutions (resp. continuous representations). We mention that also (group) characters are assumed continuous.

2. Preliminaries. In this section, we set up some notation and conventions and briefly review some fundamental facts in Fourier analysis which will be used later.

Let $d x$ denote the normalized Haar measure on $G$. Let $\hat{G}$ stand for the set of equivalence classes of continuous irreducible unitary representations of $G$. It is known that for $[\pi] \in \hat{G}, \pi$ is finite dimensional. We denote its dimension by $d_{\pi}$. Consider $\mathcal{E}_{\pi}=\operatorname{span}\left\{\pi_{i j}: i, j=1, \ldots, d_{\pi}\right\}$ the linear span of a matrix-valued representative of $[\pi]$. For $f \in L^{2}(G)$, the Fourier transform of $f$ is defined by

$$
\hat{f}(\pi)=d_{\pi} \int_{G} f(x) \pi(x)^{-1} d x \in M_{d_{\pi}}(\mathbb{C}),
$$

for all $[\pi] \in \hat{G}$, where $M_{d_{\pi}}(\mathbb{C})$ is the space of all $d_{\pi} \times d_{\pi}$ complex matrices.

As usual, left and right regular representations of $G$ on $L^{2}(G)$ are defined by

$$
\left(L_{y} f\right)(x)=f\left(y^{-1} x\right), \quad\left(R_{y} f\right)(x)=f(x y),
$$

respectively, for all $f \in L^{2}(G)$ and $x, y \in G$. A crucial property of the Fourier transform is that it converts the regular representations of $G$ into matrix multiplications.

The following identities will be useful later:

$$
\widehat{L_{y} f}(\pi)=\hat{f}(\pi) \pi(y)^{-1}, \quad \widehat{R_{y} f}(\pi)=\pi(y) \hat{f}(\pi),
$$

for all $x, y \in G$, and $\pi \in \hat{G}$.

For more information about the topics of this section, refer to [13, Chapter 5]. 
3. Main result. In this section, we solve the functional equation (1.1) by expressing its solutions in terms of unitary characters of $G$. The following lemmas derive some properties of the solutions of (1.1).

Lemma 3.1. Let $f: G \rightarrow \mathbb{C}$ be a non-zero solution of the functional equation (1.1). Then

(a) $f(e)=1$.

(b) $f \circ \sigma=f$.

Proof. (a) Setting $y=e$ in (1.1) gives us $n f(x)(f(e)-1)=0$ for all $x \in G$. Since $f \neq 0$, then $f(e)=1$.

(b) Taking $x=e$ in (1.1), we get that

$$
f(y)+\sum_{k=1}^{n-1} f\left(\sigma^{k}(y)\right)=n f(y), \quad y \in G .
$$

Interchanging $y$ and $\sigma(y)$ in the last equation, we obtain after a small computation that

$$
f(y)+\sum_{k=1}^{n-1} f\left(\sigma^{k}(y)\right)=n f(\sigma(y)), \quad y \in G .
$$

So $f(\sigma(y))=f(y)$ for all $y \in G$, i.e., $f \circ \sigma=f$.

Lemma 3.2. Let $f: G \rightarrow \mathbb{C}$ be a non-zero solution of (1.1). There exists $[\pi] \in \hat{G}$ such that $\hat{f}(\pi)$ is invertible.

Proof. Since $f \circ \sigma=f$ and $\sigma^{n}=I$, we can reformulate (1.1) to

$$
n f(x) f=L_{x^{-1}} f+\sum_{k=1}^{n-1} R_{\sigma^{n-k}(x)} f=L_{x^{-1}} f+\sum_{l=1}^{n-1} R_{\sigma^{l}(x)} f, \quad x \in G .
$$

Taking the Fourier transform to the last equation and using the identities given in Section 2, we have

$$
\hat{f}(\pi) \pi(x)+\sum_{k=1}^{n-1} \pi\left(\sigma^{k}(x)\right) \hat{f}(\pi)=n f(x) \hat{f}(\pi), \quad x \in G .
$$

Since $f \not \equiv 0$, there exists $[\pi] \in \hat{G}$ with $\hat{f}(\pi) \neq 0$. Now, let $v$ be a vector in $\operatorname{ker} \hat{f}(\pi)$. From (3.1), we infer that $\hat{f}(\pi) \pi(x) v=0$ for all $x \in G$. So $\pi(x) \operatorname{ker} \hat{f}(\pi) \subset \operatorname{ker} \hat{f}(\pi)$ for all $x \in G$. Since $\pi$ is irreducible and $\hat{f}(\pi) \neq 0$, we have $\operatorname{ker} \hat{f}(\pi)=\{0\}$. This implies that $\hat{f}(\pi)$ is bijective, thus invertible as a matrix.

With the use of the previous lemmas, we now describe the complete solution of (1.1) on an arbitrary compact group. It is clear that $f \equiv 0$ is a solution of (1.1), so in the following theorem we are only concerned with the non-zero solutions. 
Theorem 3.3. The non-zero solutions $f: G \rightarrow \mathbb{C}$ of (1.1) are the functions of the form

$$
f=\frac{1}{n} \sum_{k=0}^{n-1} \chi \circ \sigma^{k}
$$

where $\chi$ is a unitary character of $G$.

Proof. Using Lemma 3.2 and equality (3.1), we see that there exists $[\pi] \in \hat{G}$ such that

$$
\pi(x)+\sum_{k=1}^{n-1} \hat{f}(\pi)^{-1} \pi\left(\sigma^{k}(x)\right) \hat{f}(\pi)=n f(x) I_{d_{\pi}}, \quad x \in G .
$$

Taking the trace on both sides of (3.2), we obtain

$$
\operatorname{tr}(\pi(x))+\sum_{k=1}^{n-1} \operatorname{tr}\left(\pi\left(\sigma^{k}(x)\right)\right)=n d_{\pi} f(x), \quad x \in G,
$$

which abbreviates to

$$
f(x)=\frac{1}{n d_{\pi}} \sum_{k=0}^{n-1} \operatorname{tr}\left(\pi\left(\sigma^{k}(x)\right)\right), \quad x \in G .
$$

Each term on the right hand side of (3.3) is a central function, because trace is a central function. Hence $f$ is central, which implies that $\hat{f}(\pi)$ is an intertwining operator for $\pi$. But $\pi$ is irreducible, so $\hat{f}(\pi)=\mu I_{d_{\pi}}$ for some $\mu \in \mathbb{C}$ by Schur's lemma. Actually $\mu \neq 0$, because $\hat{f}(\pi) \neq 0$. Now Eq. (3.2) coalesces into

$$
\sum_{k=0}^{n-1} \pi\left(\sigma^{k}(x)\right)=n f(x) I_{d_{\pi}}, \quad x \in G .
$$

Let $(\mathcal{H} ;\langle\rangle$,$) denote the complex Hilbert space on which the representation$ $\pi$ acts, and consider the set

$$
S=\left\{k \in\{1, \ldots, n-1\} \mid \pi \simeq \pi \circ \sigma^{k}\right\} .
$$

We will consider two cases, $S$ is empty or not.

In the first case, from (3.4) we get that

$$
\pi_{i j}(x)+\sum_{k=1}^{n-1}\left(\pi\left(\sigma^{k}(x)\right)\right)_{i j}=0 \text { for } i \neq j, 1 \leq i, j \leq d_{\pi}, \quad x \in G .
$$

Since $S=\emptyset$, we have $\mathcal{E}_{\pi} \perp \mathcal{E}_{\pi \circ \sigma^{k}}$ for all $k=1, \ldots, n-1$. Hence $\pi_{i j}=0$ for $i \neq j$, so $\pi$ is a diagonal matrix. Since $\pi$ is irreducible we have $d_{\pi}=1$.

In the second case, i.e. $S \neq \emptyset$, we have

$$
S=\left\{s_{0}, 2 s_{0}, \ldots, N s_{0}\right\} \quad \text { and } n=(N+1) s_{0},
$$


where $s_{0}=\min S$ and $N=\operatorname{card} S$. Indeed, let $k \in S$, there exists $(q, r) \in$ $\mathbb{N} \times \mathbb{N}$ such that $k=q s_{0}+r$ and $0 \leq r<s_{0}$. From $\pi \simeq \pi \circ \sigma^{s_{0}}$ we arrive at $\pi \circ \sigma^{r} \simeq \pi \circ \sigma^{q s_{0}+r}$, so $\pi \circ \sigma^{r} \simeq \pi \circ \sigma^{k}$. This implies that $\pi \simeq \pi \circ \sigma^{r}$. Since $0 \leq r<s_{0}$ and $s_{0}=\min S$, we have $r=0$. Then $S$ is contained in the set of integer multiples of $s_{0}$. An additional simple inductive argument is needed to show that $S$ has the form $S=\left\{s_{0}, 2 s_{0}, \ldots, N s_{0}\right\}$. Furthermore, $\pi \simeq \pi \circ \sigma^{s_{0}}$ is equivalent to $\pi \simeq \pi \circ \sigma^{n-s_{0}}$. From $\pi \simeq \pi \circ \sigma^{n-s_{0}}$ we infer that $n-s_{0} \in S$. Since $n-s_{0}+s_{0}=n \notin S$, we see that $n-s_{0}$ is the biggest element in $S=\left\{s_{0}, 2 s_{0}, \ldots, N s_{0}\right\}$, i.e., $n-s_{0}=N s_{0}$ and hence $n=(N+1) s_{0}$. This finishes the proof of (3.5).

Since $\pi \simeq \pi \circ \sigma^{s_{0}}$, there exists a unitary operator $T$ on $\mathcal{H}$ such that

$$
\pi \circ \sigma^{s_{0}}(x)=T^{*} \pi(x) T, \quad x \in G,
$$

which by a simple induction gives us the more general formula

$$
\pi \circ \sigma^{k s_{0}}(x)=\left(T^{k}\right)^{*} \pi(x) T^{k}, \quad x \in G, k=1,2, \ldots
$$

Since $T$ is a unitary matrix, by the spectral theorem for normal operators applied to $T$, we infer that $T$ is diagonalizable. Then $\mathcal{H}$ has an orthonormal basis $\left(e_{1}, e_{2}, \ldots, e_{d_{\pi}}\right)$ consisting of eigenvectors of $T$. We write $T e_{i}=\lambda_{i} e_{i}$ where $\lambda_{i} \in \mathbb{C}$ for $i=1,2, \ldots, d_{\pi}$. Actually $\left|\lambda_{i}\right|=1$, because $T$ is unitary. For any $i=1,2, \ldots, d_{\pi}$ and $k=1,2, \ldots$, we compute that

$$
\begin{aligned}
\left(\pi \circ \sigma^{k s_{0}}(x)\right)_{i i} & =\left\langle\pi \circ \sigma^{k s_{0}}(x) e_{i}, e_{i}\right\rangle=\left\langle\left(T^{k}\right)^{*} \pi(x) T^{k} e_{i}, e_{i}\right\rangle \\
& =\left\langle\pi(x) T^{k} e_{i}, T^{k} e_{i}\right\rangle=\left\langle\lambda_{i}^{k} \pi(x) e_{i}, \lambda_{i}^{k} e_{i}\right\rangle \\
& =\lambda_{i}^{k} \overline{\lambda_{i}^{k}}\left\langle\pi(x) e_{i}, e_{i}\right\rangle=\left|\lambda_{i}\right|^{2 k} \pi_{i i}(x)=\pi_{i i}(x),
\end{aligned}
$$

for all $x \in G$ and $k \in S$. From (3.4), we infer that

$$
\pi_{i i}(x)+\sum_{k=1}^{N} \pi_{i i}\left(\sigma^{k s_{0}}(x)\right)+\sum_{k \in \bar{S}} \pi_{i i}\left(\sigma^{k}(x)\right)=n f(x),
$$

for all $i=1, \ldots, d_{\pi}$ and $x \in G$, where $\bar{S}$ denotes the complement of $S$ in $\{1, \ldots, n-1\}$. Using (3.6) and the fact that $\left(\pi \circ \sigma^{k s_{0}}\right)_{i i}=\pi_{i i}$ for $k=$ $1, \ldots, N$, we obtain

$$
(N+1) \pi_{i i}(x)+\sum_{k \in \bar{S}} \pi_{i i}\left(\sigma^{k}(x)\right)=n f(x),
$$

for all $i=1, \ldots, d_{\pi}$ and $x \in G$. Then $d_{\pi}=1$. Indeed, if $d_{\pi}>1$, then for all $i=2, \ldots, d_{\pi}$ we have

$$
(N+1) \pi_{i i}+\sum_{k \in \bar{S}} \pi_{i i} \circ \sigma^{k}=(N+1) \pi_{11}+\sum_{k \in \bar{S}} \pi_{11} \circ \sigma^{k},
$$


SO

$$
(N+1)\left(\pi_{i i}-\pi_{11}\right)=\sum_{k \in \bar{S}}\left(\pi_{11}-\pi_{i i}\right) \circ \sigma^{k} .
$$

Since $\pi$ is not equivalent to $\pi \circ \sigma^{k}$ for all $k \in \bar{S}$, we have $\mathcal{E}_{\pi} \perp \mathcal{E}_{\pi \circ \sigma^{k}}$ for all $k \in \bar{S}$. Then (3.7) implies that $\pi_{i i}=\pi_{11}$ for all $i=2, \ldots, d_{\pi}$. But if you use Schur's orthogonality relations which say $\frac{1}{d_{\pi}} \pi_{i j}$ is an orthonormal basis, we get a contradiction. Then $d_{\pi}=1$.

Finally, in view of these cases we deduce that $d_{\pi}=1$. From $d_{\pi}=1$ we see that $\pi$ is a unitary character, say $\pi=\chi$, and we deduce from (3.4) that

$$
f=\frac{1}{n} \sum_{k=0}^{n-1} \chi \circ \sigma^{k} \text {. }
$$

Conversely, a simple computation proves that the formula above for $f$ defines a solution of (1.1).

Corollary 3.4. The non-zero solutions $f: G \rightarrow \mathbb{C}$ of the functional equation (1.2) are the functions of the form

$$
f=\frac{\chi+\chi \circ \sigma}{2}
$$

where $\chi$ is a unitary character of $G$.

Acknowledgement. We wish to express our thanks to the referees for useful comments.

\section{REFERENCES}

[1] Akkouchi, M., Bouikhalene, B., Elqorachi, E., Functional equations and K-spherical functions, Georgian Math. J. 15 (2008), 1-20.

[2] An, J., Yang, D., Nonabelian harmonic analysis and functional equations on compact groups, J. Lie Theory 21 (2011), 427-455.

[3] Badora, R., On a joint generalization of Cauchy's and d'Alembert's functional equations, Aequationes Math. 43 (1992), 72-89.

[4] Chahbi, A., Fadli, B., Kabbaj, S., Functional equations of Cauchy's and d'Alembert's type on compact groups, Proyecciones (Antofagasta) 34 (2015), 297-305.

[5] Chojnacki, W., On some functional equation generalizing Cauchy's and d'Alembert's functional equations, Colloq. Math. 55 (1988), 169-178.

[6] Chojnacki, W., On group decompositions of bounded cosine sequences, Studia Math. 181 (2007), 61-85.

[7] Chojnacki, W., On uniformly bounded spherical functions in Hilbert space, Aequationes Math. 81 (2011), 135-154.

[8] Fadli, B., Zeglami, D., Kabbaj, S., A variant of Wilson's functional equation, Publ. Math. Debrecen, to appear.

[9] Davison, T. M. K., D'Alembert's functional equation on topological groups, Aequationes Math. 76 (2008), 33-53.

[10] Davison, T. M. K., D'Alembert's functional equation on topological monoids, Publ. Math. Debrecen, 75 (2009), 41-66. 
[11] de Place Friis, P., D'Alembert's and Wilson's equation on Lie groups, Aequationes Math. 67 (2004), 12-25.

[12] Elqorachi, E., Akkouchi, M., Bakali, A., Bouikhalene, B., Badora's equation on nonAbelian locally compact groups, Georgian Math. J. 11 (2004), 449-466.

[13] Folland, G., A Course in Abstract Harmonic Analysis, CRC Press, Boca Raton, FL, 1995.

[14] Shin'ya, H., Spherical matrix functions and Banach representability for locally compact motion groups, Japan. J. Math. (N.S.), 28 (2002), 163-201.

[15] Stetkær, H., D'Alembert's functional equations on metabelian groups, Aequationes Math. 59 (2000), 306-320.

[16] Stetkær, H., D'Alembert's and Wilson's functional equations on step 2 nilpotent groups, Aequationes Math. 67 (2004), 241-262.

[17] Stetkær, H., Properties of d'Alembert functions, Aequationes Math. 77 (2009), 281301.

[18] Stetkær, H., Functional Equations on Groups, World Scientfic, Singapore, 2013.

[19] Stetkær, H., D'Alembert's functional equation on groups, Banach Center Publ. 99 (2013), 173-191.

[20] Stetkær, H., A variant of d'Alembert's functional equation, Aequationes Math. 89 (2015), 657-662.

[21] Yang, D., Factorization of cosine functions on compact connected groups, Math. Z. 254 (2006), 655-674.

[22] Yang, D., Functional equations and Fourier analysis, Canad. Math. Bull. 56 (2013), 218-224.

A. Chahbi

Department of Mathematics

Faculty of Sciences

IBN TOFAIL University

BP: 14000. KENITRA

Morocco

e-mail: abdellatifchahbi@gmail.com

S. Kabbaj

Department of Mathematics

Faculty of Sciences

IBN TOFAIL University

BP: 14000. KENITRA

Morocco

e-mail: samkabbaj@yahoo.fr

Received August 20, 2015
B. Fadli

Department of Mathematics

Faculty of Sciences

IBN TOFAIL University

BP: 14000. KENITRA

Morocco

e-mail: himfadli@gmail.com 